\title{
Recycling of flap pedicle in complex lower extremity reconstruction: A proof of free muscle flap neovascularization
}

\author{
Georgios Pafitanis ${ }^{1,2}$, Alexandros Vris ${ }^{3}$, Dimitris Reissis ${ }^{2}$, Amir Sadri ${ }^{2}$, Reza Alamouti², \\ Simon Myers ${ }^{1,2}$, Parviz Sadigh ${ }^{2}$ \\ ${ }^{1}$ Group for Academic Plastic Surgery, The Blizard Institute, Barts' and The London School of Medicine and Dentistry, Queen Mary University \\ of London, London; Departments of ${ }^{2}$ Plastic Surgery and ${ }^{3}$ Orthopedics, The Royal London Hospital, Barts Health NHS Trust, London, UK
}

This article portrays the authors' clinical experience of a complex case of lower extremity reconstruction using a recycled pedicle from 10 years old free latissimus dorsi musculocutaneous flap to supply a new free anterolateral thigh flap for proximal tibia wound defect reconstruction. It provides clinical evidence that muscle neovascularization occurs and supports the dogma peripheral tissue neovascularization. This case stipulates that recycling of pedicle is feasible, when used with appropriate strategy and safety and also provides evidence for the long-term survival of greater saphenous vein grafts in lower extremity reconstruction.

Keywords Muscle neovascularization / Vein graft / Recycle flap / Flap pedicle
Correspondence: Georgios Pafitanis Group for Academic Plastic Surgery, The Blizard Institute, Barts' and The London School of Medicine and Dentistry, Queen Mary University of London, 4 Newark Street, Whitechapel, E12AT, London, UK Tel: +44-20-7882-2586 E-mail: g.pafitanis@qmul.ac.uk

Received: February 19, $2018 \bullet$ Revised: May 16, $2020 \bullet$ Accepted: June 18, 2020

pISSN: 2234-6163 • elSSN: 2234-6171 • https://doi.org/10.5999/aps.2018.00171 • Arch Plast Surg 2020;47:619-621

\section{INTRODUCTION}

The mechanism and extent of neovascularization occurring in free flaps postoperatively is yet to be proven. Until recently it was thought that neovascularization of musculocutaneous flaps occurs by angiogenesis at the peripheral skin edges, with very little new blood supply developing to the muscle itself. This dogma has been based on reported cases of partial or complete flap failure after division of the major pedicle $[1,2]$. However, a recent experimental study by Gundeslioglu et al. [3] has demonstrated that muscle flaps can survive independently of their major pedicle as soon as a week from tissue transfer, as revascularization occurs from well-nourished adjacent tissues. This is of direct clinical relevance when revision surgery is required in the vicinity of a previous free muscle flap, where the pedicle to that flap becomes compromised or is required for further anastomosis to supply a new free flap.

\section{CASE}

We report a case of recycling the pedicle of a previous free latissimus dorsi (LD) myocutaneous free flap to act as the recipient vessels for a new free anterolateral thigh (ALT) flap in the adjacent region of the same lower limb, with complete survival of both free flaps to allow definitive soft tissue reconstruction. The 43 years old patient, following informed consent, had undergone the free LD myocutaneous flap 10 years previously to cover an extensive soft tissue defect overlying concomitant GustiloAnderson Type IIIb proximal and middle third right tibial and fibula comminuted fractures, sustained following a high energy 


\section{Fig. 1. Recycled flap pedicle in lower extremity reconstruction}

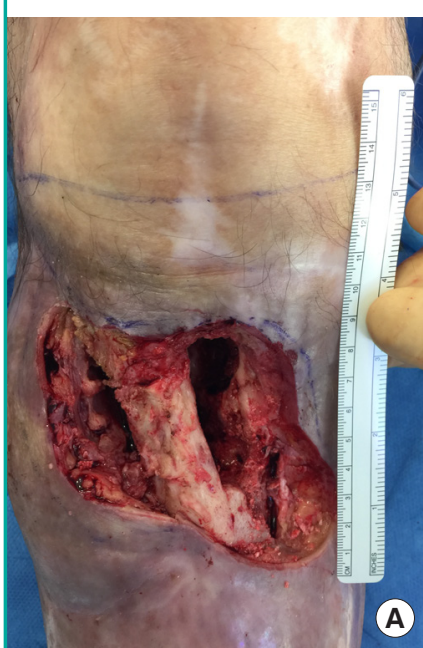

(A) Debrided proximal anterior tibia wound. (B) The latissimus dorsi (LD) muscle flap with the split-thickness skin graft (STSG; violet), anterolateral thigh (ALT)-vastus lateralis (blue) free flap with the arterial and venous pedicle vessels and the microvascular anastomoses to the saphenous vein grafts. (C) Postoperative photograph: new ALT free flap (blue) and the LD grafted muscle flap (violet) with its skin paddle (yellow). The LD pedicle transected and the ALT pedicle anastomosed to the proximally based vein grafts also demonstrated. GSV, great saphenous vein.
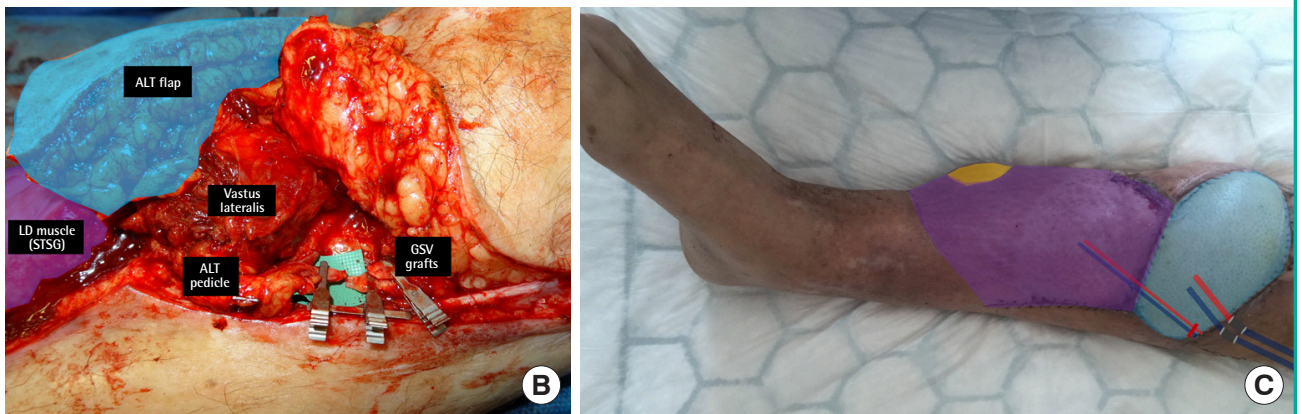

\section{Fig. 2. CTA confirming flow through GSV graft}

Computed tomography angiogram (CTA) demonstrating good filling through the vein graft, starting from the adductors hiatus and also demonstrated the level of the anastomoses with the pedicle of the new free anterolateral thigh (ALT) flap. Microvascular coupler device for great saphenous vein (GSV)-venous comitant and endto-end anastomosis vein graft-descending branch of lateral circumflex femoral artery (top left).

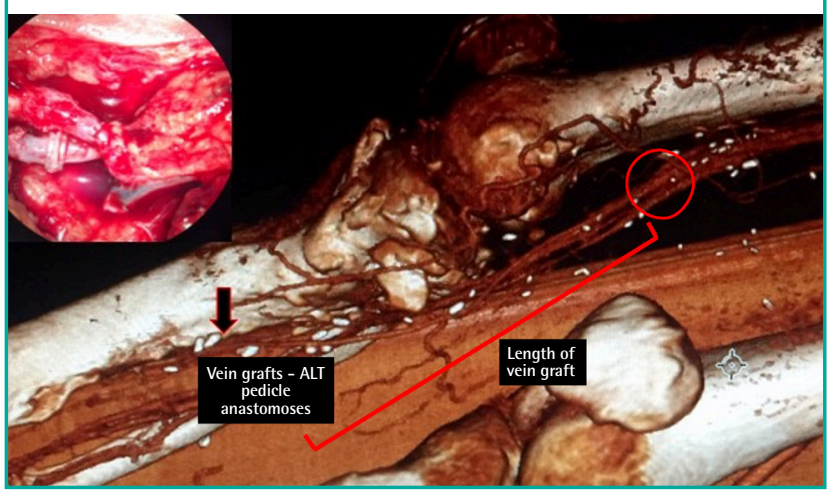

road traffic accident. The lack of loco-regional recipient vessels necessitated the use of great saphenous vein (GSV) grafts to anastomose the thoracodorsal pedicle to the recipient superficial femoral artery (SFA). Seven years postoperatively he presented with recurring blisters and discharging sinuses overlying the proximal tibia fracture site. Chronic osteomyelitis was confirmed on magnetic resonance imaging scan and treated with debridement and filling of the defect with polymethylmethacrylate spacer with gentamycin beads, leaving an overlying soft tissue defect $10 \times 15 \mathrm{~cm}$ at the level of the tibial metaphysis (Fig. 1). Computed tomography angiogram demonstrated flow through the GSV graft (caliber 3-4 mm), extending between the SFA or a large branch, such as the descending genicular artery, towards the anastomosis with the LD muscle flap pedicle, at the level of the middle third of the tibia ( $>20 \mathrm{~cm}$ ) (Fig. 2). A free ALT flap was used for definitive soft tissue reconstruction to cover the exposed fracture site. Intra-operatively proximal and inferior exploration failed to identify pulsatile anterior tibial or medial genicular arteries. The previously used GSV grafts remained patent and robust following surgical dissection. A microclamp perfusion test, to both inflow and outflow, confirmed neovascularization of the LD flap via peripheral perfusion of its skin paddle, which was surrounded by split-thickness skin graft LD muscle. Thus, a decision was taken to recycle this GSV graft pedicle as the recipient vessels for the new free ALT flap [4]. Arterial anastomosis was performed using 8-0 nylon suture between the GSV graft (from the SFA) and the donor descending lateral circumflex femoral artery. Venous anastomosis was performed using a $4.0 \mathrm{~mm}$ coupler between the second GSV graft (to the femoral vein) to the venae comitentes of the descending lateral circumflex femoral artery. The patient made a full recovery with robust soft tissue coverage and survival of both free flaps (Supplementary Figs. 1, 2).

\section{DISCUSSION}

This case demonstrates a clinical proof of neovascularization of a musculocutaneous LD free flap. Thus, its major supplying pedicle could be effectively recycled to act as recipient and successfully supply a new free flap. We also note that this relies on the continued patency and 10-year survival of this high-flow GSV large caliber graft, which could adequately re-perfuse the newly transferred free ALT flap. Vlastou et al. [5] demonstrated the value and efficacy of long vein grafting, with either the short or GSVs, for lower extremity reconstruction. Precisely, demonstrate that when local recipient vessels are not suitable, vein 
grafts may provide the only solution. This study further reports that an average length of $20 \mathrm{~cm}$ (range, 3-30 cm) was proven reliable, and their success appear to be unrelated to the length of the venous system; and the flap survival is highly related to the severity of the trauma.

The nature and mechanism of this neovascularization of muscle free tissue transfer is not yet clear and requires further trials investigating the nature of neo-vessel angiogenesis, both from the underlying bed and the wound edges. It is essential to establish the basic science behind muscle flap neovascularization and understand the time-frame in which it occurs, as such cases provide salvage options for recycling old free flap pedicles to successfully supply new free flaps required for similar cases of chronic osteomyelitis or repeat trauma in the same affected limb.

\section{NOTES}

\section{Conflict of interest}

No potential conflict of interest relevant to this article was reported.

\section{Ethical approval}

The study was performed in accordance with the principles of the Declaration of Helsinki. Written informed consent was obtained.

\section{Patient consent}

The patient provided written informed consent for the publication and the use of his images.

\section{Author contribution}

Conceptualization: Georgios P, Alexandros V, Simon M, Parviz S. Data curation: Georgios P, Dimitris R. Formal analysis: Georgios P, Simon M. Methodology: Georgios P, Alexandros V, Amir R. Project administration: Georgios P, Alexandros V, Dimitris R. Visualization: Georgios P. Writing - original draft: all authors. Writing - review \& editing: all authors. Approval of final manuscript: all authors.

\section{ORCID}

Georgios Pafitanis

Alexandros Vris

https://orcid.org/0000-0003-4712-277X

Parviz Sadigh

https://orcid.org/0000-0001-9723-9533

https://orcid.org/0000-0003-2872-5129

\section{Supplementary material}

Supplementary Fig. 1. Immediate postoperative outcome photography. Postoperative on-table photograph demonstrating the free anterolateral thigh skin paddle, the free latissimus dorsi muscle covered with split-thickness skin graft and its skin paddle. Supplemental data can be found at: https://doi.org/10.5999/ aps.2018.00171.

Supplementary Fig. 2. Day 7 postoperative outcome photograph. Postoperative day 7 photograph of the reconstructed lower limb. Supplemental data can be found at: https://doi.org/10.5999/ aps.2018.00171.

\section{REFERENCES}

1. Manrique OJ, Chen SH, Ciudad P, et al. Neovascularization failure of an interposed free intestinal colon flap 10 years after esophageal reconstruction. J Reconstr Microsurg Open 2017;2:e35-6.

2. Burns A, Avery BS, Edge CJ. Survival of microvascular free flaps in head and neck surgery after early interruption of the vascular pedicle. Br J Oral Maxillofac Surg 2005;43:426-7.

3. Gundeslioglu AO, Selimoglu N, Toy H, et al. Neo-vascularisation of musculocutaneous and muscle flaps after division of the major vascular supply: an experimental study. J Plast Reconstr Aesthet Surg 2013;66:978-86.

4. de Vries MR, Simons KH, Jukema JW, et al. Vein graft failure: from pathophysiology to clinical outcomes. Nat Rev Cardiol 2016;13:451-70.

5. Vlastou C, Earle AS, Jordan R. Vein grafts in reconstructive microsurgery of the lower extremity. Microsurgery 1992; 13:234-5. 


\section{Supplementary Fig. 1. Immediate postoperative outcome} photography

Postoperative on-table photograph demonstrating the free anterolateral thigh skin paddle, the free latissimus dorsi muscle covered with split-thickness skin graft and its skin paddle.

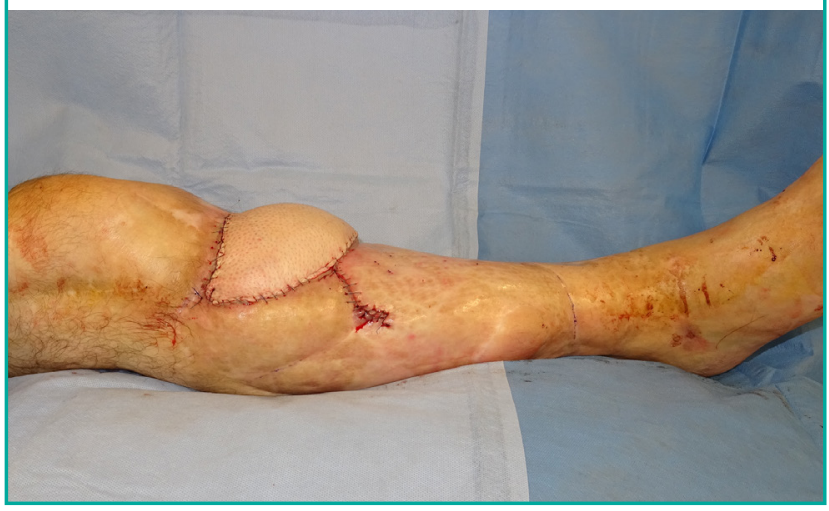

\section{Supplementary Fig. 2. Day 7 postoperative outcome} photograph

Postoperative day 7 photograph of the reconstructed lower limb.

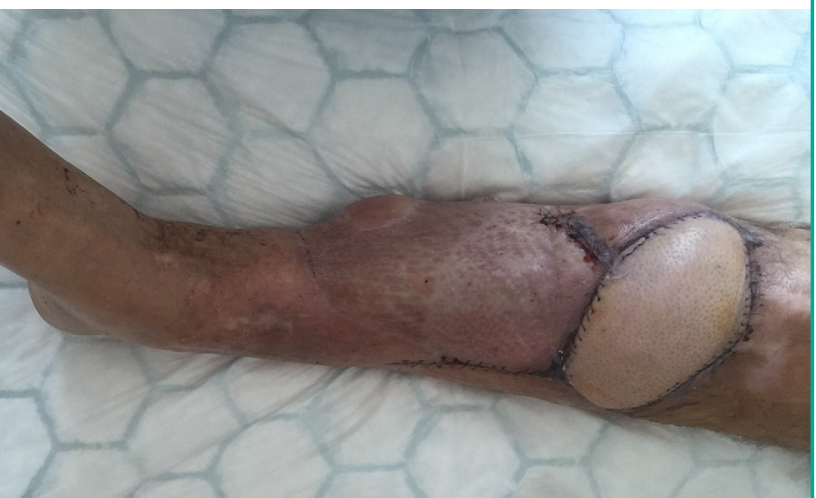

